\title{
GLOBAL STABILITY IN CHEMOSTAT-TYPE EQUATIONS WITH DISTRIBUTED DELAYS*
}

\author{
XUE-ZHONG $\mathrm{HE}^{\dagger}$, SHIGUI RUAN $\ddagger$, AND HUAXING XIA ${ }^{\S}$
}

\begin{abstract}
We consider a chemostat-type model in which a single species feeds on a limiting nutrient supplied at a constant rate. The model incorporates a general nutrient uptake function and two distributed (infinite) delays. The first delay models the fact that the nutrient is partially recycled after the death of the biomass by bacterial decomposition, and the second delay indicates that the growth of the species depends on the past concentration of the nutrient. By constructing appropriate Liapunov-like functionals, we obtain sufficient conditions for local and global stability of the positive equilibrium of the model. Quantitative estimates on the size of the delays for local and global stability are also obtained with the help of the Liapunov-like functionals. The technique we use in this paper may be used as well to study global stability of other types of physical models with distributed delays.
\end{abstract}

Key words. chemostat-type equations, distributed delay, Liapunov functionals, local and global stability, nutrient recycling

AMS subject classifications. 34K15, 34K20, 45J05, 92A15

\section{PII. S0036141096311101}

1. Introduction. The effect of material (nutrient) recycling on ecosystem stability has been previously studied for closed systems (see Nisbet and Gurney [16], Nisbet, McKinstry, and Gurney [17], and Ulanowicz [22]). Powell and Richerson [18] and Nisbet and Gurney [16] regarded nutrient recycling as an instantaneous process, thus neglecting the time required to regenerate the nutrient from the dead biomass by bacterial decomposition. However, as pointed out in Whittaker [23], a delay in nutrient recycling is always present in a natural system and it increases when temperature decreases. To simulate the growth of planktonic communities of unicellular algae in the lakes, Beretta, Bischi, and Solimano [1] proposed an open system in which a single species feeds on a limiting nutrient supplied at a constant rate. They assumed that the nutrient is partially recycled after the death of the organisms and used a distributed delay to model nutrient recycling. Bischi [5] observed that the delay involved in nutrient recycling alone does not have a destabilizing effect on the equilibrium.

Evidence of delayed growth response has also been observed from chemostat experiments with microalgae Chlamidomonas Reinhardii even when the limiting nutrient is at undetectable small concentration (see Caperon [7]). Following Caperon [7], Ruan [19] introduced a discrete delay to the model of Beretta, Bischi, and Solimano [1] to describe the delayed growth response of the species to nutrient uptake. It is shown (see He and Ruan [11]) that the positive equilibrium is globally stable if the delays

* Received by the editors October 23, 1996; accepted for publication (in revised form) April 25, 1997.

http://www.siam.org/journals/sima/29-3/31110.html

${ }^{\dagger}$ School of Mathematics and Statistics, University of Sydney, Sydney, NSW 2006, Australia (he_t@maths.su.oz.au).

${ }^{\ddagger}$ Department of Mathematics, Statistics and Computing Science, Dalhousie University, Halifax, Nova Scotia, Canada B3H 3J5 (ruan@mscs.dal.ca). The research of this author was supported by the NSERC of Canada and the Petro-Canada Young Innovator Award.

$\S$ Department of Mathematics and Statistics, McMaster University, Hamilton, Ontario, Canada L8S 4K1 (xia@icarus.math.mcmaster.ca). 
are sufficiently small. However, there is a threshold value of the discrete delay involved in the growth response; when the discrete delay is increased and passes this critical value, the equilibrium may lose its stability and a Hopf bifurcation may occur (see Ruan [19]). Recently, Beretta and Takeuchi [2-4] used an additional distributed delay to model the delayed growth response. By assuming that the response function is either a Lotka-Volterra function or a Michaelis-Menten function, they studied the global stability of the positive equilibrium.

In this paper, we consider a chemostat-type model with (distributedly) delayed growth response and (distributedly) delayed nutrient recycling, namely, a system of two retarded functional differential equations with two distributed delays. This model was first proposed and studied by Beretta and Takeuchi [2]. However, their stability results are only local. Although global stability was also considered in [2-4] for similar models with an additional instantaneous negative feedback or without delayed growth response at all, the problem is more difficult to study when delayed growth response is introduced. By constructing appropriate Liapunov-like functionals, we study both local and global stability of the positive equilibrium of the model. It turns out that the positive equilibrium can be globally asymptotically stable if the mean delays are sufficiently small, and quantitative estimates on the size of these delays can be obtained with the help of the Liapunov-like functionals. Moreover, our approach to the local stability problem is slightly different than that used by Beretta and Takeuchi [2], and we improve their local stability result.

We remark that distributed (infinite) delay equations have been used in biological modeling since the work of Volterra (see Scudo and Ziegler [20]) and they are regarded to be more realistic than discrete (finite) delay equations (see Caperon [7]). The fundamental theory and some properties such as stability, existence of periodic solutions, etc. of distributed delay equations are well understood now and are discussed in the books of Burton [6], Hale and Verduyn Lunel [10], and Hino, Murakami and Naito [12]. The monographs of Cushing [8] and MacDonald [15] give excellent descriptions of distributed delay models and study the local stability, bifurcation, and periodic solutions of these models. Although global stability of some biological models with distributed delays has been studied (see Gopalsamy [9], Kuang [14], Wolkowicz, Xia, and Ruan [24] and the references cited therein), in general global results for models involving distributed delays are hard to obtain. The reason probably is that there are few methods available in investigating the global stability of infinite delay equations. The most powerful and most important method is perhaps the Liapunov function(al) method. However, there is no general procedure to follow in constructing a desirable Liapunov function(al), and completely different forms of Liapunov function(al)s are used for different kinds of equations. In the present paper, we try to construct the Liapunov-like functionals step by step so that the idea and technique can be easily followed. We believe that our technique can be used as well to study global stability of some other types of physical models with infinite delays.

The paper is organized as follows. In section 2 we describe the model equations. Local stability is studied in section 3 and global stability is discussed in section 4 . Finally, a brief discussion is carried out in section 5 .

2. The model. Let $N(t)$ denote the limiting nutrient concentration and $P(t)$ denote the plankton concentration at time $t$. Consider the following integrodifferential equations model of plankton-nutrient interaction with delayed growth response and delayed nutrient recycling: 


$$
\begin{aligned}
& \dot{N}=D\left(N^{0}-N\right)-a U(N) P+b \gamma \int_{0}^{\infty} f(s) P(t-s) d s \\
& \dot{P}=P\left[-(\gamma+D)+c \int_{0}^{\infty} g(s) U(N(t-s)) d s\right],
\end{aligned}
$$

with initial value conditions

$$
N(\theta)=\phi_{1}(\theta) \geq 0, \quad P(\theta)=\phi_{2}(\theta) \geq 0, \quad \theta \in(-\infty, 0],
$$

where $\phi_{1}(\theta), \phi_{2}(\theta) \in B C(-\infty, 0]$, the Banach space of all continuous bounded functions, and all parameters are positive constants. $N^{0}$ is the input concentration of the limiting nutrient, $a$ is the maximum uptake rate of nutrient, $c(\leq a)$ is the maximum specific growth rate of plankton, $b(0<b<1)$ is the fraction of the nutrient recycled by bacterial decomposition of the dead plankton, $\gamma$ is the death rate of plankton, and $D$ is the washout rate, so $\gamma+D$ represents the total loss rate of the plankton.

The function $U(N)$ describes the nutrient uptake rate of plankton. Throughout, we assume that $U(N)$ is nonnegative, increasing, and vanishes when there is no nutrient, and there is a saturation effect when the nutrient is very abundant. That is, we assume that $U(N)$ is a continuously differentiable function defined on $[0, \infty)$ and

$$
U(0)=0, \quad \frac{d U}{d N}>0, \quad \lim _{N \rightarrow \infty} U(N)=1 .
$$

These general hypotheses are satisfied by the Michaelis-Menten function (see [21])

$$
U(N)=\frac{N}{L+N},
$$

where $L>0$ is the half-saturation constant or Michaelis-Menten constant.

The delay kernels $f(s)$ and $g(s)$ are nonnegative bounded functions defined on $[0, \infty) . f(s)$ describes the contribution of the plankton population dead in the past to the nutrient recycled and $g(s)$ describes the delayed growth response of plankton to nutrient uptake. The presence of the distributed time delays must not affect the equilibrium values, so we normalize the kernels such that

$$
\int_{0}^{\infty} f(s) d s=\int_{0}^{\infty} g(s) d s=1 .
$$

As in MacDonald [15], we define the average time delays as

$$
T_{f}=\int_{0}^{\infty} s f(s) d s, \quad T_{g}=\int_{0}^{\infty} s g(s) d s .
$$

Note that $E_{0}=\left(N^{0}, 0\right)$ is always an equilibrium for system $(2.1)$, and if

$$
\gamma+D<c \text { and } U^{-1}\left(\frac{\gamma+D}{c}\right)<N^{0}
$$

system (2.1) has a positive interior equilibrium $E^{*}=\left(N^{*}, P^{*}\right)$ with

$$
N^{*}=U^{-1}\left(\frac{\gamma+D}{c}\right), \quad P^{*}=\frac{D\left(N^{0}-N^{*}\right)}{a U\left(N^{*}\right)-b \gamma} .
$$

Throughout, we always assume that (2.4) is satisfied and $T_{f}$ and $T_{g}$ are finite. 
Denote by $X(t, \phi)=(N(t, \phi), P(t, \phi))$ the solution of system (2.1) satisfying the initial value conditions $(2.2)$, where $\phi=\left(\phi_{1}, \phi_{2}\right)$. We say that the positive equilibrium $E^{*}=\left(N^{*}, P^{*}\right)$ of $(2.1)$ is (locally) stable if for any $\varepsilon>0$ there exists $\delta=\delta(\varepsilon)$ such that $\left|X(t, \phi)-E^{*}\right|<\varepsilon$ for $t \geq 0$ and $\phi \in B\left(E^{*}, \delta\right)$, where $B\left(E^{*}, \delta\right)$ is an open $\delta$-ball of $E^{*} . E^{*}$ is said to be (locally) asymptotically stable if it is (locally) stable and there is a $\delta_{o}>0$ such that $\phi \in B\left(E^{*}, \delta_{o}\right)$ implies $X(t, \phi) \rightarrow E^{*}$ as $t \rightarrow \infty$. $E^{*}$ is said to be globally asymptotically stable if it is (locally) asymptotically stable, and for any positive solution $X(t, \phi)$ of $(2.1)$ and $(2.2)$, we have $X(t, \phi) \rightarrow E^{*}$ as $t \rightarrow \infty$.

System (2.1) was introduced and studied by Beretta and Takeuchi $[2,4]$. It was shown there that all solutions of system (2.1) are nonnegative if the initial data chosen from $B C(-\infty, 0]$ are nonnegative. They also discussed stability of the positive equilibrium $E^{*}$. However, their stability results about system (2.1) are only local, and global stability results hold only for systems (similar to (2.1)) with an additional instantaneous negative feedback or without delayed growth response at all. The object of this paper is to improve their local stability result and investigate as well the global stability of the positive equilibrium.

3. Local asymptotic stability. We first study the local stability of the positive equilibrium $E^{*}=\left(N^{*}, P^{*}\right)$. Let

$$
x_{1}=N-N^{*}, x_{2}=P-P^{*},
$$

where $-N^{*} \leq x_{1}<\infty,-P^{*} \leq x_{2}<\infty$, and define

$$
\xi\left(x_{1}\right)=U(N)-U\left(N^{*}\right)
$$

so that $-U\left(N^{*}\right) \leq \xi\left(x_{1}\right)<1-U\left(N^{*}\right)$ by assumption (2.3). Then the linearized equations about $E^{*}$ are

$$
\begin{aligned}
& \dot{x}_{1}=-\left(D+a P^{*} U^{\prime}\left(N^{*}\right)\right) x_{1}-a U\left(N^{*}\right) x_{2}+b \gamma \int_{0}^{\infty} f(s) x_{2}(t-s) d s, \\
& \dot{x}_{2}=c P^{*} U^{\prime}\left(N^{*}\right) \int_{0}^{\infty} g(s) x_{1}(t-s) d s .
\end{aligned}
$$

Note that the asymptotic stability of the trivial equilibrium $x_{1}=x_{2}=0$ of (3.2) implies the local asymptotic stability of the positive equilibrium $E^{*}$ of (2.1). For convenience, we define

$$
A=D+a P^{*} U^{\prime}\left(N^{*}\right), \quad B=a c P^{*} U\left(N^{*}\right) U^{\prime}\left(N^{*}\right), \quad C=b \gamma c P^{*} U^{\prime}\left(N^{*}\right) .
$$

Then $B>C$, and system (3.2) becomes

$$
\begin{aligned}
& \dot{x}_{1}=-A x_{1}-\frac{B}{C} b \gamma x_{2}+b \gamma \int_{0}^{\infty} f(s) x_{2}(t-s) d s, \\
& \dot{x}_{2}=\frac{C}{b \gamma} \int_{0}^{\infty} g(s) x_{1}(t-s) d s .
\end{aligned}
$$

Let $\left(x_{1}(t), x_{2}(t)\right)$ be an arbitrary solution of system (3.4). We first consider the function $V_{11}(t)=x_{1}^{2}(t)$. It follows from (3.4) that 


$$
\begin{aligned}
\dot{V}_{11}(t)= & 2 x_{1}(t) \dot{x}_{1}(t) \\
= & -2 A x_{1}^{2}(t)-2 b \gamma \frac{B}{C} x_{1}(t) x_{2}(t)+2 b \gamma x_{1}(t) x_{2}(t) \\
& -2 b \gamma x_{1}(t) \int_{0}^{t} f(s) \int_{t-s}^{t} \dot{x}_{2}(u) d u d s+I(t) \\
= & -2 A x_{1}^{2}(t)-2 b \gamma\left(\frac{B}{C}-1\right) x_{1}(t) x_{2}(t)+I(t) \\
& -2 C x_{1}(t) \int_{0}^{t} f(s) \int_{t-s}^{t} \int_{0}^{\infty} g(v) x_{1}(u-v) d v d u d s \\
\leq & -2 A x_{1}^{2}(t)-2 b \gamma\left(\frac{B}{C}-1\right) x_{1}(t) x_{2}(t)+I(t) \\
& +C \int_{0}^{\infty} f(s) \int_{t-s}^{t} \int_{0}^{\infty} g(v)\left[x_{1}^{2}(t)+x_{1}^{2}(u-v)\right] d v d u d s \\
= & -2 A x_{1}^{2}(t)-2 b \gamma\left(\frac{B}{C}-1\right) x_{1}(t) x_{2}(t)+C T_{f} x_{1}^{2}(t) \\
& +C \int_{0}^{\infty} f(s) \int_{t-s}^{t} \int_{0}^{\infty} g(v) x_{1}^{2}(u-v) d v d u d s+I(t),
\end{aligned}
$$

where

$$
I(t)=-2 b \gamma x_{1}(t) \int_{t}^{\infty} f(s)\left(x_{2}(t)-x_{2}(t-s)\right) d s .
$$

For technical reasons, we assume that $\int_{0}^{\infty} s^{2} f(s) d s<\infty$. Then the function

$$
V_{12}(t)=C \int_{0}^{\infty} f(s) \int_{t-s}^{t} \int_{r}^{t} \int_{0}^{\infty} g(v) x_{1}^{2}(u-v) d v d u d r d s
$$

is well defined, and by (3.5), we have

$$
\begin{aligned}
\dot{V}_{11}(t)+\dot{V}_{12}(t) \leq & -2 A x_{1}^{2}(t)-2 b \gamma\left(\frac{B}{C}-1\right) x_{1}(t) x_{2}(t)+C T_{f} x_{1}^{2}(t) \\
& +C \int_{0}^{\infty} f(s) \int_{t-s}^{t} \int_{0}^{\infty} g(v) x_{1}^{2}(t-v) d v d u d s+I(t) \\
= & -2 A x_{1}^{2}(t)-2 b \gamma\left(\frac{B}{C}-1\right) x_{1}(t) x_{2}(t)+C T_{f} x_{1}^{2}(t) \\
& +C T_{f} \int_{0}^{\infty} g(s) x_{1}^{2}(t-s) d s+I(t) .
\end{aligned}
$$

We now consider the function

$$
V_{1}(t)=V_{11}(t)+V_{12}(t)+C T_{f} \int_{0}^{\infty} g(s) \int_{t-s}^{t} x_{1}^{2}(u) d u d s .
$$

It follows from (3.7) that

$$
\dot{V}_{1}(t) \leq-2\left(A-C T_{f}\right) x_{1}^{2}(t)-2 b \gamma\left(\frac{B}{C}-1\right) x_{1}(t) x_{2}(t)+I(t) .
$$

On the other hand, by the second equation of (3.4), we have

$$
\frac{d}{d t}\left[x_{2}+\frac{C}{b \gamma} \int_{0}^{\infty} g(s) \int_{t-s}^{t} x_{1}(u) d u d s\right]=\frac{C}{b \gamma} x_{1}(t) .
$$


Assume $\int_{0}^{\infty} s^{2} g(s) d s<\infty$ and define

$$
V_{2}(t)=\left[x_{2}+\frac{C}{b \gamma} \int_{0}^{\infty} \int_{t-s}^{t} g(s) x_{1}(u) d u\right]^{2}+\left(\frac{C}{b \gamma}\right)^{2} \int_{0}^{\infty} g(s) \int_{t-s}^{t} \int_{v}^{t} x_{1}^{2}(u) d u d v d s .
$$

We find that

$$
\dot{V}_{2}(t) \leq \frac{2 C}{b \gamma} x_{1}(t) x_{2}(t)+2\left(\frac{C}{b \gamma}\right)^{2} T_{g} x_{1}^{2}(t) .
$$

Therefore, for the function

$$
V(t)=V_{1}(t)+\frac{(b \gamma)^{2}}{C}\left(\int_{t}^{\infty} f(s) d s+\frac{B}{C}-1\right) V_{2}(t),
$$

we have from (3.6), (3.8), and (3.9) that

$$
\begin{aligned}
\dot{V}(t) \leq & -2\left[A-C T_{f}-\left(C \int_{t}^{\infty} f(s) d s+B-C\right) T_{g}\right] x_{1}^{2}(t) \\
& +2 b \gamma x_{1}(t) x_{2}(t) \int_{t}^{\infty} f(s) d s+I(t) \\
= & -2\left[A-C T_{f}-\left(C \int_{t}^{\infty} f(s) d s+B-C\right) T_{g}\right] x_{1}^{2}(t) \\
& +2 b \gamma x_{1}(t) \int_{t}^{\infty} f(s) x_{2}(t-s) d s \\
\leq & -2\left[A-C T_{f}-\left(C \int_{t}^{\infty} f(s) d s+B-C\right) T_{g}\right] x_{1}^{2}(t) \\
& +2 b \gamma\left|x_{1}(t)\right|\left\|\phi_{2}\right\| \int_{t}^{\infty} f(s) d s \\
\leq & -2\left[A-C T_{f}-\left(C \int_{t}^{\infty} f(s) d s+B-C\right) T_{g}\right] x_{1}^{2}(t) \\
& +b \gamma x_{1}^{2}(t) \int_{t}^{\infty} f(s) d s+b \gamma\left\|\phi_{2}\right\|^{2} \int_{t}^{\infty} f(s) d s \\
= & -2\left[A-C T_{f}-(B-C) T_{g}\right] x_{1}^{2}(t) \\
& +\left(2 C T_{g}+b \gamma\right) x_{1}^{2}(t) \int_{t}^{\infty} f(s) d s+b \gamma\left\|\phi_{2}\right\|^{2} \int_{t}^{\infty} f(s) d s
\end{aligned}
$$

where $\phi_{2} \in B C(-\infty, 0]$ is the initial data of $x_{2}(t)$. By using (3.10), we now can prove the following local stability result.

THEOREM 3.1. Assume that $\int_{0}^{\infty} s^{2} f(s) d s<\infty$ and $\int_{0}^{\infty} s^{2} g(s) d s<\infty$. If

$$
C T_{f}+(B-C) T_{g}<A,
$$

then the positive equilibrium $E^{*}$ of system (2.1) is locally asymptotically stable.

Proof. Let $\left(x_{1}(t), x_{2}(t)\right)$ be an arbitrary solution of (3.4) with $\phi_{2} \in B C(-\infty, 0]$ being the initial data for $x_{2}(t)$. By (3.11), we can find $\varepsilon>0$ such that

$$
Q(\varepsilon) \triangleq C T_{f}+(B-C) T_{g}+\left(C T_{g}+\frac{1}{2} b \gamma\right) \varepsilon<A .
$$


Let $T=T(\varepsilon)>0$ be such that $\int_{t}^{\infty} f(s) d s<\varepsilon$ for all $t \geq T$. It then follows from (3.10) that for all $t \geq T$,

$$
\dot{V}(t) \leq-2(A-Q(\varepsilon)) x_{1}^{2}(t)+b \gamma\|\phi\|^{2} \int_{t}^{\infty} f(s) d s .
$$

Integrating $\dot{V}(t)$ from $T$ to $t \geq T$ gives

$$
\begin{aligned}
x_{1}^{2}(t) & +\frac{(b \gamma)^{2}}{C^{2}}(B-C) V_{2}(t)+2(A-Q(\varepsilon)) \int_{T}^{t} x_{1}^{2}(s) d s \\
& \leq V(t)+2(A-Q(\varepsilon)) \int_{T}^{t} x_{1}^{2}(s) d s \\
& \leq V(T)+b \gamma\left\|\phi_{2}\right\|^{2} \int_{T}^{t} \int_{s}^{\infty} f(u) d u d s \\
& \leq V(T)+b \gamma\left\|\phi_{2}\right\|^{2} \int_{0}^{\infty} s f(s) d s \\
& =V(T)+b \gamma\left\|\phi_{2}\right\|^{2} T_{f}<\infty .
\end{aligned}
$$

Therefore, $x_{1}(t)$ and $x_{2}(t)$ are bounded, and $x_{1}^{2}(t) \in L_{1}[0, \infty)$. By the mean value theorem and the equations in (3.4), $x_{1}(t), x_{2}(t)$, and their derivative functions are thus uniformly continuous on $[0, \infty)$. Applying the Barbălat lemma (see Lemmas 1.2.2 and 1.2.3 in Gopalsamy [9]), we conclude that $\left(x_{1}(t), \dot{x}_{1}(t)\right) \rightarrow 0$ as $t \rightarrow \infty$. Therefore, from the first equation of (3.4), we must have

$$
\lim _{t \rightarrow \infty}\left[-\frac{B}{C} x_{2}(t)+\int_{0}^{\infty} f(s) x_{2}(t-s) d s\right]=0 .
$$

Let $\alpha=\liminf _{t \rightarrow \infty} x_{2}(t), \beta=\limsup _{t \rightarrow \infty} x_{2}(t)$, and $\left\{t_{m}\right\} \uparrow \infty$ be a sequence such that $x_{2}\left(t_{m}\right) \rightarrow \beta$ as $m \rightarrow \infty$. Then $\beta<\infty$, and from (3.12) we obtain

$$
\frac{B}{C} \beta=\lim _{m \rightarrow \infty} \int_{0}^{\infty} f(s) x_{2}\left(t_{m}-s\right) d s \leq \beta .
$$

Since $B>C$, this implies that $\beta \leq 0$. A similar argument shows that $\alpha \geq 0$. Therefore $\alpha=\beta=0$, and $\left(x_{1}(t), x_{2}(t)\right) \rightarrow(0,0)$ as $t \rightarrow \infty$ for every solution $\left(x_{1}(t), x_{2}(t)\right)$ of system (3.4).

Note that the characteristic equation of (3.4) is

$$
\Delta(\lambda)=\lambda^{2}+A \lambda+G(\lambda)(B-C F(\lambda))=0,
$$

where

$$
F(\lambda)=\int_{0}^{\infty} f(s) e^{-\lambda s} d s, \quad G(\lambda)=\int_{0}^{\infty} g(s) e^{-\lambda s} d s .
$$

Since $B>C$ and every solution of (3.4) approaches zero as $t \rightarrow \infty, \Delta(\lambda)$ has no roots with $\operatorname{Re}(\lambda) \geq 0$. Therefore, all roots of $\Delta(\lambda)$ have negative real parts and $E^{*}$ is thus locally asymptotically stable. This completes the proof.

Remark 3.2. Beretta and Takeuchi $[2,4]$ observed that system (3.4) has the same characteristic equation as the following system:

$$
\begin{aligned}
& \dot{y}_{1}=y_{2}, \\
& \dot{y}_{2}=-A y_{2}-\int_{0}^{\infty} B g(s) y_{1}(t-s) d s+C \int_{0}^{\infty} \int_{0}^{\infty} g(s-v) f(v) y_{1}(t-s) d v d s .
\end{aligned}
$$


They then constructed a Liapunov functional for (3.13) and showed that the sufficient condition for the local stability of $E^{*}$ is (Theorem 2 in [4])

$$
C T_{f}+(B+C) T_{g}<A .
$$

Our condition (3.11) improves the above condition.

4. Global asymptotic stability. To study the global stability of $E^{*}$, we consider any positive solution $X(t, \phi)=(N(t, \phi), P(t, \phi))$ of $(2.1)$ and (2.2). We make the change of variables

$$
x_{1}=N-N^{*}, \quad x_{2}=\ln \left(P / P^{*}\right),
$$

and define $\xi\left(x_{1}\right)$ as in (3.1). Then

$$
N=x_{1}+N^{*}, \quad P=P^{*} \exp \left(x_{2}\right),
$$

and $x_{1} \xi\left(x_{1}\right)>0$ for any $x_{1} \in\left[-N^{*},+\infty\right) ; x_{1} \xi\left(x_{1}\right)=0$ if and only if $x_{1}=0$. Using (4.1), we rewrite system (2.1) as follows:

$$
\begin{aligned}
\dot{x}_{1}(t)= & -D x_{1}(t)-a P^{*} \exp \left(x_{2}(t)\right) \xi\left(x_{1}(t)\right)-P^{*} G\left(\exp \left(x_{2}(t)\right)-1\right) \\
& -b \gamma P^{*} \int_{0}^{t} \int_{t-s}^{t} f(s) \exp \left(x_{2}(u)\right) \dot{x}_{2}(u) d u d s+J(t) \\
\dot{x}_{2}(t)= & c \int_{0}^{\infty} g(s) \xi\left(x_{1}(t-s)\right) d s
\end{aligned}
$$

where $G=a U\left(N^{*}\right)-b \gamma>0$ and

$$
J(t)=-b \gamma P^{*} \int_{t}^{\infty} f(s)\left[\exp \left(x_{2}(t)\right)-\exp \left(x_{2}(t-s)\right)\right] d s .
$$

Let $\left(x_{1}(t), x_{2}(t)\right)$ be an arbitrary solution of system (4.3). We consider the function

$$
V_{11}(t)=\int_{0}^{x_{1}(t)} \xi(s) d s .
$$

Then upon using (4.3), we obtain

$$
\begin{aligned}
\dot{V}_{11}(t)= & -D x_{1}(t) \xi\left(x_{1}(t)\right)-a P(t) \xi^{2}\left(x_{1}(t)\right)-P^{*} G\left(\exp \left(x_{2}(t)\right)-1\right) \xi\left(x_{1}(t)\right) \\
& -b \gamma P^{*} \xi\left(x_{1}(t)\right) \int_{0}^{t} \int_{t-s}^{t} f(s) \exp \left(x_{2}(u)\right) \dot{x}_{2}(u) d u d s+\xi\left(x_{1}(t)\right) J(t) \\
= & -D x_{1}(t) \xi\left(x_{1}(t)\right)-a P(t) \xi^{2}\left(x_{1}(t)\right) \\
& -P^{*} G\left(\exp \left(x_{2}(t)\right)-1\right) \xi\left(x_{1}(t)\right)+\xi\left(x_{1}(t)\right) J(t) \\
& -b c \gamma P^{*} \xi\left(x_{1}(t)\right) \int_{0}^{t} \int_{t-s}^{t} f(s) \exp \left(x_{2}(u)\right) \int_{0}^{\infty} g(v) \xi\left(x_{1}(u-v)\right) d v d u d s \\
\leq & -D x_{1}(t) \xi\left(x_{1}(t)\right)-a P(t) \xi^{2}\left(x_{1}(t)\right)-P^{*} G\left(\exp \left(x_{2}(t)\right)-1\right) \xi\left(x_{1}(t)\right) \\
& +\frac{1}{2} b c \gamma\left(\int_{0}^{\infty} \int_{t-s}^{t} f(s) P(u) d u d s\right) \xi^{2}\left(x_{1}(t)\right)+\xi\left(x_{1}(t)\right) J(t) \\
& +\frac{1}{2} b c \gamma \int_{0}^{\infty} \int_{t-s}^{t} f(s) P(u) \int_{0}^{\infty} g(v) \xi^{2}\left(x_{1}(u-v)\right) d v d u d s .
\end{aligned}
$$


Let us now consider the following two functions:

$$
\begin{aligned}
& V_{12}(t)=\frac{1}{2} b c \gamma \int_{0}^{\infty} f(s) \int_{t-s}^{t} \int_{w}^{t} P(u) \int_{0}^{\infty} g(v) \xi^{2}\left(x_{1}(u-v)\right) d v d u d w d s \\
& V_{13}(t)=\frac{1}{2} b c \gamma T_{f} \int_{0}^{\infty} g(s) \int_{t-s}^{t} P(u+s) \xi^{2}\left(x_{1}(u)\right) d u d s .
\end{aligned}
$$

We now assume that $\int_{0}^{\infty} s^{2} f(s) d s<\infty$ so that $V_{12}$ is well defined. To see the existence of $V_{13}$, we note from (2.1) that

$$
\dot{P}(t) \leq P(t)[c-(\gamma+D)]=k P(t)
$$

with $k=c-(\gamma+D)>0$. This implies

$$
P(s) \leq P(t) e^{k(s-t)} \quad \text { for } \quad s \geq t \geq 0 .
$$

Since $|\xi(x)| \leq 1$, using (4.7), we have from the definition of $V_{13}$ that

$$
\begin{aligned}
V_{13}(t) & \leq \frac{1}{2} b c \gamma T_{f} P(t) \int_{0}^{\infty} g(s) \int_{t-s}^{t} e^{k(u+s-t)} d u d s \\
& =\frac{1}{2 k} b c \gamma T_{f} P(t) \int_{0}^{\infty} g(s)\left[e^{k s}-1\right] d s .
\end{aligned}
$$

It follows that if $\int_{0}^{\infty} g(s)\left[e^{k s}-1\right] d s<\infty$, then $V_{13}(t)$ exists. Thus, for $V_{1}(t)=$ $V_{11}(t)+V_{12}(t)+V_{13}(t)$, we obtain from $(4.5)$ that

$$
\begin{aligned}
\dot{V}_{1}(t) \leq & -D x_{1}(t) \xi\left(x_{1}(t)\right)-a P(t) \xi^{2}\left(x_{1}(t)\right)-P^{*} G\left(\exp \left(x_{2}(t)\right)-1\right) \xi\left(x_{1}(t)\right) \\
& +\frac{1}{2} b c \gamma \xi^{2}\left(x_{1}(t)\right) \int_{0}^{\infty} \int_{t-s}^{t} f(s) P(u) d u d s \\
& +\frac{1}{2} b c \gamma T_{f} \xi^{2}\left(x_{1}(t)\right) \int_{0}^{\infty} g(s) P(t+s) d s+\xi\left(x_{1}(t)\right) J(t) .
\end{aligned}
$$

Notice that from the second equation of system $(2.1)$, we have $\dot{P}(t) \geq-(\gamma+D) P(t)$ for all $t>0$. Thus

$$
P(s) \leq P(t) \exp [(\gamma+D)(t-s)] \text { for } t \geq s \geq 0
$$

This implies that

$$
\begin{aligned}
\int_{0}^{\infty} \int_{t-s}^{t} f(s) P(u) d u d s= & \int_{0}^{t} \int_{t-s}^{t} f(s) P(u) d u d s+\int_{t}^{\infty} \int_{0}^{t} f(s) P(u) d u d s+K(t) \\
\leq & P(t) \int_{0}^{t} \int_{t-s}^{t} f(s) \exp [(\gamma+D)(t-u)] d u d s \\
& +P(t) \int_{t}^{\infty} \int_{0}^{t} f(s) \exp [(\gamma+D)(t-u)] d u d s+K(t) \\
\leq & \frac{P(t)}{\gamma+D} \int_{0}^{\infty} f(s)(\exp [(\gamma+D) s]-1) d s+K(t) \\
= & T_{f}^{*} P(t)+K(t),
\end{aligned}
$$


where

$$
\begin{aligned}
& K(t)=\int_{t}^{\infty} f(s) \int_{t-s}^{0} P(u) d u d s, \\
& T_{f}^{*}=\frac{1}{\gamma+D} \int_{0}^{\infty} f(s)(\exp [(\gamma+D) s]-1) d s .
\end{aligned}
$$

Similarly, using (4.10), we have

$$
\int_{0}^{\infty} g(s) P(t+s) d s \leq P(t) \int_{0}^{\infty} g(s) \exp [k s] d s=\left(1+k T_{g}^{*}\right) P(t)
$$

where

$$
T_{g}^{*}=\frac{1}{k} \int_{0}^{\infty} g(s)\left[e^{k s}-1\right] d s<\infty .
$$

Then (4.9), together with (4.11) and (4.14), implies that

$$
\begin{aligned}
\dot{V}_{1}(t) \leq & -D x_{1}(t) \xi\left(x_{1}(t)\right)-a P(t) \xi^{2}\left(x_{1}(t)\right) \\
& -P^{*} G\left(\exp \left(x_{2}(t)\right)-1\right) \xi\left(x_{1}(t)\right) \\
& +\frac{1}{2} b c \gamma\left(T_{f}^{*}+T_{f}+k T_{f} T_{g}^{*}\right) P(t) \xi^{2}\left(x_{1}(t)\right) \\
& +\xi\left(x_{1}(t)\right) J(t)+\frac{1}{2} b c \gamma \xi^{2}\left(x_{1}(t)\right) K(t) .
\end{aligned}
$$

On the other hand, from the second equation of system (4.3), we have

$$
\frac{d}{d t}\left[x_{2}(t)+c \int_{0}^{\infty} g(s) \int_{t-s}^{t} \xi\left(x_{1}(u)\right) d u d s\right]=c \xi\left(x_{1}(t)\right) .
$$

Let

$$
y(t)=x_{2}(t)+c \int_{0}^{\infty} g(s) \int_{t-s}^{t} \xi\left(x_{1}(u)\right) d u d s .
$$

We define

$$
V_{21}(t)=\int_{0}^{y(t)}[\exp (s)-1] d s
$$

Then it follows that

$$
\begin{aligned}
\dot{V}_{21}(t)= & c \xi\left(x_{1}(t)\right)\left\{\exp \left[x_{2}(t)+c \int_{0}^{\infty} g(s) \int_{t-s}^{t} \xi\left(x_{1}(u)\right) d u d s\right]-1\right\} \\
= & c \xi\left(x_{1}(t)\right)\left[\exp \left(x_{2}(t)\right)-1\right] \\
& +c \xi\left(x_{1}(t)\right)\left\{\exp \left[x_{2}(t)+c \int_{0}^{\infty} g(s) \int_{t-s}^{t} \xi\left(x_{1}(u)\right) d u d s\right]-\exp \left(x_{2}(t)\right)\right\} \\
= & c \xi\left(x_{1}(t)\right)\left[\exp \left(x_{2}(t)\right)-1\right] \\
& +c \exp \left(x_{2}(t)\right) \xi\left(x_{1}(t)\right)\left\{\exp \left[c \int_{0}^{\infty} g(s) \int_{t-s}^{t} \xi\left(x_{1}(u)\right) d u d s\right]-1\right\} \\
= & c \xi\left(x_{1}(t)\right)\left[\exp \left(x_{2}(t)\right)-1\right] \\
& +c^{2} \exp \left(x_{2}(t)\right) \xi\left(x_{1}(t)\right) \exp (\alpha(t)) \int_{0}^{\infty} g(s) \int_{t-s}^{t} \xi\left(x_{1}(u)\right) d u d s
\end{aligned}
$$


for some function $\alpha(t)$ between 0 and $c \int_{0}^{\infty} g(s) \int_{t-s}^{t} \xi\left(x_{1}(u)\right) d u d s$. Since $\left|\xi\left(x_{1}\right)\right| \leq 1$, we have

$$
|\alpha(t)| \leq c \int_{0}^{\infty} g(s) \int_{t-s}^{t}\left|\xi\left(x_{1}(u)\right)\right| d u d s \leq c T_{g}
$$

Therefore,

$$
\begin{aligned}
\dot{V}_{21}(t) \leq & c \xi\left(x_{1}(t)\right)\left[\exp \left(x_{2}(t)\right)-1\right] \\
& +\frac{1}{2} c^{2} \exp \left(x_{2}(t)\right) \exp (\alpha(t)) \int_{0}^{\infty} \int_{t-s}^{t} g(s)\left[\xi^{2}\left(x_{1}(t)\right)+\xi^{2}\left(x_{1}(u)\right)\right] d u d s \\
= & c \xi\left(x_{1}(t)\right)\left[\exp \left(x_{2}(t)\right)-1\right] \\
& +\frac{1}{2} c^{2} \exp \left(x_{2}(t)\right) \exp (\alpha(t))\left[T_{g} \xi^{2}\left(x_{1}(t)\right)+\int_{0}^{\infty} \int_{t-s}^{t} g(s) \xi^{2}\left(x_{1}(u)\right) d u d s\right] \\
\leq & c \xi\left(x_{1}(t)\right)\left[\exp \left(x_{2}(t)\right)-1\right] \\
& +\frac{1}{2} c^{2} T_{g} \exp \left(x_{2}(t)\right) \exp \left(c T_{g}\right) \xi^{2}\left(x_{1}(t)\right) \\
& +\frac{1}{2} c^{2} \exp \left(x_{2}(t)\right) \exp \left(c T_{g}\right) \int_{0}^{\infty} \int_{t-s}^{t} g(s) \xi^{2}\left(x_{1}(u)\right) d u d s .
\end{aligned}
$$

We now define

$$
V_{22}(t)=\frac{1}{2} c^{2} \exp \left(c T_{g}\right) \int_{0}^{\infty} g(s) \int_{t-s}^{t} \exp \left(x_{2}(v+s)\right) \int_{v}^{t} \xi^{2}\left(x_{1}(u) d u d v d s .\right.
$$

Then, by using (4.7) and

$$
\begin{aligned}
\int_{0}^{\infty} g(s) & \int_{t-s}^{t} P(v+s) \int_{v}^{t} d u d v d s \\
& \leq \int_{0}^{\infty} s g(s) \int_{t-s}^{t} P(v+s) d v d s \\
& \leq P(t) \int_{0}^{\infty} s g(s) \int_{t-s}^{t} e^{k(v+s-t)} d v d s=\frac{1}{k} P(t) \int_{0}^{\infty} s g(s)\left[e^{k s}-1\right] d s
\end{aligned}
$$

we can see that, under the assumption that $\int_{0}^{\infty} s g(s)\left[e^{k s}-1\right] d s<\infty, V_{22}(t)$ exists. Let $V_{2}(t)=V_{21}(t)+V_{22}(t)$; we have from (4.17) that

$$
\begin{aligned}
\dot{V}_{2}(t) \leq & c \xi\left(x_{1}(t)\right)\left[\exp \left(x_{2}(t)\right)-1\right] \\
& +\frac{1}{2} c^{2} T_{g} \exp \left(c T_{g}\right) \exp \left(x_{2}(t)\right) \xi^{2}\left(x_{1}(t)\right) \\
& +\frac{1}{2} c^{2} \exp \left(c T_{g}\right) \xi^{2}\left(x_{1}(t)\right) \int_{0}^{\infty} g(s) \int_{t-s}^{t} \exp \left(x_{2}(u+s)\right) d u d s .
\end{aligned}
$$

Note that from (4.7) we have

$$
\int_{t-s}^{t} \exp \left(x_{2}(u+s)\right) d u \leq \frac{1}{k}\left[e^{k s}-1\right] \exp \left(x_{2}(t)\right) .
$$


Therefore,

$$
\begin{aligned}
\dot{V}_{2}(t) \leq & c \xi\left(x_{1}(t)\right)\left[\exp \left(x_{2}(t)\right)-1\right] \\
& +\frac{1}{2} c^{2} \exp \left(c T_{g}\right)\left(T_{g}+T_{g}^{*}\right) \exp \left(x_{2}(t)\right) \xi^{2}\left(x_{1}(t)\right) .
\end{aligned}
$$

We finally define the following function:

$$
V(t)=V_{1}(t)+\frac{P^{*}}{c}\left(b \gamma \int_{t}^{\infty} f(s) d s+G\right) V_{2}(t) .
$$

It then follows from (4.16) and (4.18) that

$$
\begin{aligned}
\dot{V}(t) \leq & -D x_{1}(t) \xi\left(x_{1}(t)\right)-\frac{1}{2}(2 a-L) P(t) \xi^{2}\left(x_{1}(t)\right) \\
& +\frac{1}{2} b c \gamma P^{*} \exp \left(c T_{g}\right)\left(T_{g}+T_{g}^{*}\right) \exp \left(x_{2}(t)\right) \xi^{2}\left(x_{1}(t)\right) \int_{t}^{\infty} f(s) d s \\
& +b \gamma P^{*} \xi\left(x_{1}(t)\right) \int_{t}^{\infty} f(s)\left[\exp \left(x_{2}(t-s)\right)-1\right] d s \\
& +\frac{1}{2} b c \gamma \xi^{2}\left(x_{1}(t)\right) K(t),
\end{aligned}
$$

where

$$
L=b c \gamma\left(T_{f}^{*}+T_{f}+k T_{f} T_{g}^{*}\right)+c G \exp \left(c T_{g}\right)\left(T_{g}+T_{g}^{*}\right) .
$$

Notice that from (4.2), $P(u)=P^{*} \exp \left(\phi_{2}(u)\right)$ for $u \leq 0$ and $\exp \left(x_{2}(t-s)\right)=$ $\exp \left(\phi_{2}(t-s)\right)$ for $t \leq s$, where $\phi_{2} \in B C(-\infty, 0]$ is the initial data for $x_{2}(t)$. By (4.12), we have

$$
\begin{aligned}
& |K(t)| \leq P^{*} \exp \left(\left\|\phi_{2}\right\|\right) \int_{t}^{\infty} s f(s) d s \\
& \left|\int_{t}^{\infty} f(s)\left[\exp \left(x_{2}(t-s)\right)-1\right] d s\right| \leq\left[\exp \left(\left\|\phi_{2}\right\|\right)-1\right] \int_{t}^{\infty} f(s) d s .
\end{aligned}
$$

Since $\left|\xi\left(x_{1}\right)\right| \leq 1$, using the above inequalities, we obtain from (4.19) that

$$
\begin{aligned}
\dot{V}(t) \leq & -D x_{1}(t) \xi\left(x_{1}(t)\right)-\frac{1}{2}(2 a-L) P(t) \xi^{2}\left(x_{1}(t)\right) \\
& +\frac{1}{2} b c \gamma \exp \left(c T_{g}\right)\left(T_{g}+T_{g}^{*}\right) P(t) \xi^{2}\left(x_{1}(t)\right) \int_{t}^{\infty} f(s) d s \\
& +b \gamma P^{*}\left[\exp \left(\left\|\phi_{2}\right\|\right)-1\right] \int_{t}^{\infty} f(s) d s \\
& +\frac{1}{2} b c \gamma P^{*} \exp \left(\left\|\phi_{2}\right\|\right) \int_{t}^{\infty} s f(s) d s .
\end{aligned}
$$

The above analysis now leads to the following global stability result.

THEOREM 4.1. Let $G=a U\left(N^{*}\right)-b \gamma$ and $T_{f}^{*}$ and $T_{g}^{*}$ be the constants defined in (4.13) and (4.15), respectively. Assume that $\int_{0}^{\infty} s g(s)\left[e^{k s}-1\right] d s<\infty$ and

$$
L \triangleq b c \gamma\left(T_{f}^{*}+T_{f}+k T_{f} T_{g}^{*}\right)+c G \exp \left(c T_{g}\right)\left(T_{g}+T_{g}^{*}\right)<2 a
$$


with $k=c-(\gamma+D)>0$. Then the positive equilibrium $E^{*}$ is globally asymptotically stable.

Proof. Let $\left(x_{1}(t), x_{2}(t)\right)$ be an arbitrary solution of system (4.3) with $\phi_{2} \in$ $B C(-\infty, 0]$ being the initial data of $x_{2}(t)$. We choose $\varepsilon>0$ such that

$$
L(\varepsilon) \triangleq L+\frac{1}{2} b c \gamma \exp \left(c T_{g}\right)\left(T_{g}+T_{g}^{*}\right) \varepsilon<2 a
$$

and find $T=T(\varepsilon)>1$ such that $\int_{t}^{\infty} f(s) d s<\varepsilon$ for all $t \geq T$. Notice that $L<2 a$ and $\int_{0}^{\infty} s^{2} g(s)\left[e^{k s}-1\right] d s<\infty$, thus $V(t)$ is well defined and (4.20) holds. Therefore, for all $t \geq T$, we have

$$
\begin{aligned}
\dot{V}(t) \leq & -D x_{1}(t) \xi\left(x_{1}(t)\right)-\frac{1}{2}(2 a-L(\varepsilon)) P(t) \xi^{2}\left(x_{1}(t)\right) \\
& +\frac{1}{2} b \gamma P^{*}\left[(c+2) \exp \left(\left\|\phi_{1}\right\|\right)-2\right] \int_{t}^{\infty} s f(s) d s \\
\leq & -D x_{1}(t) \xi\left(x_{1}(t)\right)+M \int_{t}^{\infty} s f(s) d s
\end{aligned}
$$

where $M=\frac{1}{2} b \gamma P^{*}\left[(c+2) \exp \left(\left\|\phi_{1}\right\|\right)-2\right]>0$. Integrating $\dot{V}(t)$ from $T$ to $t \geq T$ now gives

$$
\begin{aligned}
& V_{11}(t)+\frac{P^{*} G}{C} V_{2}(t)+D \int_{T}^{t} x_{1}(s) \xi\left(x_{1}(s)\right) d s \\
& \quad \leq V(t)+D \int_{T}^{t} x_{1}(s) \xi\left(x_{1}(s)\right) d s \\
& \quad \leq V(T)+M \int_{T}^{t} \int_{s}^{\infty} u f(u) d u d s \\
& \quad \leq V(T)+M \int_{0}^{\infty} s^{2} f(s) d s \\
& \quad \leq V(T)+2(\gamma+D) M T_{f}^{*}<\infty .
\end{aligned}
$$

This implies that $x_{1}(t)$ and $x_{2}(t)$ are bounded, and $x_{1}(t) \xi\left(x_{1}(t)\right) \in L_{1}[0, \infty)$. Since $\xi$ is uniformly continuous on $[0, \infty)$, it follows from the equations in system (4.3) and the boundedness of $x_{1}(t)$ and $x_{2}(t)$ that $x_{1}(t) \xi\left(x_{1}(t)\right)$ is also uniformly continuous. Thus, by the Barbălat lemma (see Lemma 1.2.2 in [9]), $x_{1}(t) \xi\left(x_{1}(t)\right) \rightarrow 0$ as $t \rightarrow \infty$. This leads to $\lim _{t \rightarrow \infty} x_{1}(t)=0$, so $\lim _{t \rightarrow \infty} N(t)=N^{*}$ by (4.2). Note further that $\dot{x}_{1}(t)$ is also uniformly continuous on $[0, \infty)$ by the equations in (4.3). Applying the Barbălat lemma (see Lemma 1.2.3 in [9]) once again gives $\lim _{t \rightarrow \infty} \dot{x}_{1}(t)=\lim _{t \rightarrow \infty} \dot{N}(t)=0$. Now taking the limit $(t \rightarrow \infty)$ on both sides of $(2.1)$, we obtain

$$
\lim _{t \rightarrow \infty}\left(a U\left(N^{*}\right) P(t)-b \gamma \int_{0}^{\infty} f(s) P(t-s) d s\right)=D\left(N^{0}-N^{*}\right) .
$$

Let $\alpha=\liminf _{t \rightarrow \infty} P(t), \beta=\lim \sup _{t \rightarrow \infty} P(t)$, and $\left\{t_{m}\right\} \uparrow \infty$ be a sequence such that $\lim _{m \rightarrow \infty} P\left(t_{m}\right)=\beta$. Since $\beta<\infty$, it follows from (4.22) that

$$
\begin{aligned}
a U\left(N^{*}\right) \beta & =D\left(N^{0}-N^{*}\right)+\lim _{m \rightarrow \infty} b \gamma \int_{0}^{\infty} f(s) P\left(t_{m}-s\right) d s \\
& \leq D\left(N^{0}-N^{*}\right)+b \gamma \beta .
\end{aligned}
$$


Thus,

$$
\beta \leq \frac{D\left(N^{0}-N^{*}\right)}{a U\left(N^{*}\right)-b \gamma}=P^{*} .
$$

Similarly, we can show that $\alpha \geq P^{*}$. Therefore, $\lim _{t \rightarrow \infty} P(t)=P^{*}$. This proves the global attractivity of $E^{*}$.

On the other hand, we claim that $L<2 a$ implies (3.11). In fact,

$$
C T_{f}+(B-C) T_{g}=c P^{*} U^{\prime}\left(N^{*}\right)\left[b \gamma T_{f}+G T_{g}\right]
$$

with $G=a U\left(N^{*}\right)-b \gamma>0$. It is noticed that $T_{f} \leq T_{f}^{*}$ and $T_{g} \leq T_{g}^{*}$. Then, from $L<2 a$, we have

$$
2 a>b c \gamma\left(T_{f}^{*}+T_{f}\right)+c G\left(T_{g}+T_{g}^{*}\right) \geq 2 c\left[b \gamma T_{f}+G T_{g}\right]
$$

and hence

$$
C T_{f}+(B-C) T_{g} \leq P^{*} U^{\prime}\left(N^{*}\right) a<A,
$$

which shows that (3.11) holds. By Theorem $3.1, E^{*}$ is locally asymptotically stable. This, together with global attractivity, implies that $E^{*}$ is globally asymptotically stable.

5. Discussion. In this paper, we have considered a chemostat-type plankton model with nutrient recycling. We assumed that there are a (distributed) delay in the growth response of plankton to nutrient uptake and a (distributed) delay in nutrient recycling. We have obtained some sufficient conditions for both local and global stability of the positive equilibrium by constructing appropriate Liapunov-like functionals. It is known that the delay in the growth response of the populations to nutrient uptake can cause oscillations in population density (see Caperon [7] and Ruan [19]); our results indicate that one can still have global stability of the positive equilibrium if the delays are sufficiently small, and explicit estimates (see (3.11), (4.18), and (4.19)) on the size of these delays for local and global stability can also be obtained.

We should point out that model (2.1) and other related models have been studied by Beretta and Takeuchi [2-4], He and Ruan [11], and Kolmanovskii, Torelli, and Vermiglio [13]. For local stability, following the arguments of Kolmanovskii, Torelli, and Vermiglio [13], Beretta and Takeuchi [2, 4] chose a Liapunov functional for the equivalent system (3.13) and obtained some sufficient conditions. Our local stability conditions improve their conditions. For global stability, Beretta and Takeuchi $[2,4]$ considered the special cases of model (2.1) when the second delay does not appear, that is, the system (2.1) with Dirac delta function $g(s)=\delta(s)$. In [2], they assumed that the interaction between nutrient and biotic species is described by (no-delayed) LotkaVolterra coupling (i.e., $U(N)=N)$. In [4], they adopted a (no-delayed) MichaelisMenten law (i.e., $U(N)=N /(L+K)$ ), which is better than the former to describe the interaction from the biological point of view. With this choice, they proved (Theorem 7 in [4]) that the positive equilibrium $E^{*}$ is attractive if

$$
\gamma T_{f}<\min \left\{\frac{1}{b}, \frac{2}{b} \sqrt{\frac{a D N^{*}}{c^{2} U\left(N^{*}\right) K}}\right\} \leq \frac{1}{b},
$$

where $K=\max \left\{\beta, N^{o} /\left(1-b \gamma T_{f}\right)\right\}, \beta=\left(1+b \gamma T_{f}\right) H$, and $H$ is the bound for initial functions. Hence, the attractivity of $N^{*}$ is indeed for all the solutions whose initial 
functions are bounded by $H$. Obviously, $\gamma T_{f} \rightarrow 0$ as $H \rightarrow \infty$. If we consider Beretta and Takeuchi's model in [4] as a special case of our system (2.1), then we have $T_{g}=$ $T_{g}^{*}=0$ and our global asymptotic stability condition in Theorem 4.1 becomes

$$
b c \gamma\left[T_{f}^{*}+T_{f}\right]<2 a .
$$

Condition (5.2) was also obtained in He and Ruan [11, Theorem 2.1] for the system $(2.1)$ with $g(s)=\delta(s)$ and the general growth response $U(N)$. Rewrite (5.2) as

$$
\gamma T_{f}+\frac{1}{2} \gamma\left[T_{f}^{*}-T_{f}\right]<\frac{a}{b c}
$$

Note that $c \leq a$, so $a / b c \geq 1 / b$. Compared with (5.1), we can see that (5.3) is more restrictive on the delay kernel function $f$ but less restrictive on the initial functions. Since condition (5.1) depends on the bound of the initial values, basically it is not a global stability condition. Thus, our condition (5.3) complements Beretta and Takeuchi's results in [4] by providing really global stability results with a little more restriction on the delay. Furthermore, our global stability results hold for the general case when both distributed delays are present and the growth response function is a general function satisfying (2.3).

Acknowledgments. Conditions in Theorem 4.1 were improved by following the referees' suggestions. We thank both referees for their comments. We are also grateful to Wanbiao Ma for his careful reading of the original version of the paper and for his helpful comments and suggestions.

\section{REFERENCES}

[1] E. Beretta, G. I. Bischi, And F. Solimano, Stability in chemostat equations with delayed nutrient recycling, J. Math. Biol., 28 (1990), pp. 99-111.

[2] E. Beretta And Y. TAKeuchi, Qualitative properties of chemostat equations with time delays: Boundedness, local and global asymptotic stability, Differential Equations Dynam. Systems, 2 (1994), pp. 19-40.

[3] E. BERETTA AND Y. TAKEUCHI, Qualitative properties of chemostat equations with time delays II, Differential Equations Dynam. Systems, 2 (1994), pp. 263-288.

[4] E. Beretta AND Y. TAKeUChI, Global stability for chemostat equations with delayed nutrient recycling, Nonlinear World, 1 (1994), pp. 291-306.

[5] G. I. BISCHI, Effects of time lags on transient characteristics of a nutrient cycling model, Math. Biosci., 109 (1992), pp. 151-175.

[6] T. A. Burton, Volterra Integral and Differential Equations, Academic Press, New York, 1983.

[7] J. CAPERON, Time lag in population growth response of isochrysis galbana to a variable nitrate environment, Ecology, 50 (1969), pp. 188-192.

[8] J. M. Cushing, Integrodifferential Equations and Delay Models in Population Dynamics, Springer-Verlag, Heidelberg, 1977.

[9] K. Gopalsamy, Stability and Oscillations in Delay Differential Equations of Population Dynamics, Kluwer Academic Publishers, Dordrecht, The Netherlands, 1992.

[10] J. K. Hale and S. M. Verduyn Lunel, Introduction to Functional Differential Equations, Appl. Math. Sci. 99, Springer-Verlag, New York, 1993.

[11] X.-Z. He And S. Ruan, Global Stability in Chemostat-Type Plankton Models with Delayed Nutrient Recycling, Research report 96-8, The School of Math. \& Stat., University of Sydney, 1996.

[12] Y. Hino, S. Murakami, And T. Naito, Functional Differential Equations with Infinite Delay, Lecture Notes in Math. 1473, Springer-Verlag, New York, 1991.

[13] V. B. Kolmanovskit, L. Torelli, and R. Vermiglio, Stability of some test equations with delay, SIAM J. Math. Anal., 25 (1994), pp. 948-961.

[14] Y. Kuang, Delay Differential Equations with Applications in Population Dynamics, Academic Press, New York, 1993. 
[15] N. MacDonald, Time Lags in Biological Models, Springer-Verlag, Heidelberg, 1978.

[16] R. M. Nisbet AND W. S. C. GuRney, Model of material cycling in a closed ecosystem, Nature, 264 (1976), pp. 633-635.

[17] R. M. Nisbet, J. McKinstry, And W. S. C. Gurney, A strategic model of material cycling in a closed ecosystem, Math. Biosci., 64 (1983), pp. 99-113.

[18] T. POWEll AND P. J. Richerson, Temporal variation, spatial heterogeneity and competition for resource in plankton system: A theoretical model, Amer. Nat., 125 (1985), pp. 431-464.

[19] S. RuAN, The effect of delays on stability and persistence in plankton models, Nonlinear Analysis, 24 (1995), pp. 575-585.

[20] F. M. Scudo AND J. R. Ziegler, The Golden Age of Theoretical Ecology: 1923-1940, Lecture Notes in Biomathematics 22, Springer-Verlag, Berlin, 1978.

[21] H. L. Smith And P. Waltman, The Theory of the Chemostat, Cambridge University Press, Cambridge, 1994.

[22] R. E. Ulanowicz, Mass and energy flow in closed ecosystems, J. Theor. Biol., 34 (1972), pp. 239-253.

[23] R. H. Whittaker, Communities and Ecosystems, Macmillan, New York, 1975.

$[24]$ G. S. K. Wolkowicz, H. XIA, And S. RuAn, Competition in the chemostat: A distributed delay model and its global asymptotic behavior, SIAM J. Appl. Math., 57 (1997), pp. 12811310 . 